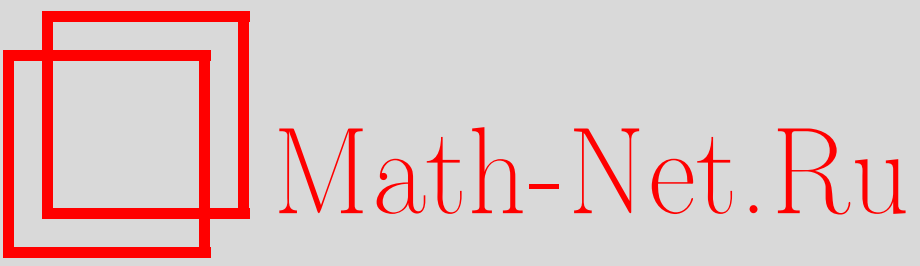

О. А. Индюков, М. А. Попов, О высокотемпературной функции релаксации спиновой системы при квадратичном вкладе флуктуаций в резонансную частоту, TMФ, 1999, том 121, номер 2, 316-328

DOI: https://doi.org/10.4213/tmf812

Использование Общероссийского математического портала Math-Net.Ru подразумевает, что вы прочитали и согласны с пользовательским соглашением

http://www.mathnet.ru/rus/agreement

Параметры загрузки:

IP : 3.85 .5 .30

26 апреля 2023 г., $15: 35: 25$ 


\section{О ВЫСОКОТЕМПЕРАТУРНОЙ ФУНКЦИИ РЕЛАКСАЦИИ СПИНОВОЙ СИСТЕМЫ ПРИ КВАДРАТИЧНОМ ВКЛАДЕ ФЛУКТУАЦИЙ В РЕЗОНАНСНУЮ ЧАСТОТУ}

Исследуется форма высокотемпературной функции релаксации спиновой системы при квадратичной связи резонансной частоты с гауссовским случайным процессом. В общем случае она приведена к интегральному выражению от бесконечного вспомогательного ряда. Для $N$-экспоненциального гаусс-марковского процесса задача сведена к решению системы из $2 N$ линейных уравнений. Анализ действия фолуктуаций для компак тности выполнен на форме линии магнитного резонанса - фурье-образе функции релаксации. Как для одноэкспоненциального процесса, так и для многоэкспоненциального в кристалле с релаксационным типом динамики в области непрерывного фазового перехода выявлена немонотонная зависимость асимметричной однородно уширенной резонансной линии от скорости фолуктуаций.

\section{ВВЕДЕНИЕ}

Высокая чувствительность частоты магнитного резонанса $\Omega$ к величине магнитного поля на резонируюшем спине широко используется [1] для исследования в конденсированных средах как локальной структуры, так и подвижности атомов и их спинов, вызывающей флуктуации $\Omega$. Связанная преобразованием Фурье с формой резонансной линии и наблюдаемая при импульсном воздействии на спиновую систему образца, функция релаксации (спад свободной прецессии) в приближении Андерсона $[2,3]$ задается соотношением

$$
G(t)=\left\langle\exp \left\{-i \int_{0}^{t} \Omega\left(t_{1}\right) d t_{1}\right\}\right\rangle,
$$

где угловые скобки обозначают усреднение по испытываемым резонансной частотой флуктуациям. При выводе (1) для спиновой системы использовалось высокотемпературное приближение (приближение Ван-Флека) и полагалось пренебрежимо малым взаимодействие, приводящее к переходам между задаваемыми сильным постоянным магнитным полем энергетическими уровнями (адиабатическое приближение).

* Красноярский государственный университет, Красноярск, Россия. E-mail: root@iph.krasnoyarsk.su 
Для объяснения многих эффектов в спиновой динамике: сужения резонансной линии обменным взаимодействием [2] и тепловыми фолуктуациями [3], спинового эха [4], крыльев спектров [5] и т.п. - успешным оказался учет в резонансной частоте линейного вклада флуктуаций (назовем их решеточными) $\Omega(t)=\Omega_{0}+\Omega_{1} \eta(t)$, описываемых в случае высокой плотности и наличия дальнодействия в решеточной системе образца стационарным гауссовским случайным процессом с $\langle\eta\rangle=0$. Однако во многих случаях действие фллуктуаций не может быть сведено к линейному вкладу, например, в спиновых системах с обменным взаимодействием выше билинейного [6] или в системах, облучаемых сильным радиочастотным полем [7]. Кроме того, отсутствие нечетных слагаемых в разложении $\Omega$ по $\eta$ характерно для спинов, находящихся в частных позициях ячейки кристалла. Поэтому в данной работе действие таких флуктуаций на высокотемпературную функцию релаксации спиновой системы исследуется при их квадратичном вкладе в резонансную частоту, когда $\Omega(t)=\Omega_{0}+\Omega_{1} \eta(t)+\Omega_{2} \eta(t)^{2} / 2$.

\section{1. ОБший подХод}

Представим функцию релаксации в виде ряда

$$
G(t)=\exp \left\{-i \Omega_{0} t\right\} \sum_{n=0}^{\infty} \frac{(-i)^{n}}{n !}\left\langle\left\{\int_{0}^{t}\left[\Omega_{1} \eta\left(t_{1}\right)+\frac{\Omega_{2} \eta\left(t_{1}\right)^{2}}{2}\right] d t_{1}\right\}^{n}\right\rangle
$$

Для расчета $n$-го слагаемого в (2) обратимся к $n$-мерной характеристической функции

$$
\Phi_{n}\{\lambda\} \equiv \Phi\left(\lambda_{1}, \lambda_{2}, \ldots, \lambda_{n}\right)=\prod_{k=1}^{n}\left\{\int_{-\infty}^{\infty} \exp \left(i \lambda_{k} \eta\left(t_{k}\right)\right) d \eta\left(t_{k}\right)\right\} P_{n}\{\eta\}
$$

где $P_{n}\{\eta\} \equiv P\left(\eta\left(t_{1}\right), \eta\left(t_{2}\right), \ldots, \eta\left(t_{n}\right)\right)-n$-мерная плотность вероятности случайного процесса. Знание $\Phi_{n}\{\lambda\}$ позволяет найти среднее произведения $k$ решеточных переменных при $k \leqslant n$ :

$$
\left\langle\eta_{1}^{m_{1}} \eta_{2}^{m_{2}} \ldots \eta_{k}^{m_{k}}\right\rangle=\left.i^{-n_{k}} \frac{\partial^{n_{k}} \Phi_{n}}{\partial \lambda_{1}^{m_{1}} \partial \lambda_{2}^{m_{2}} \ldots \partial \lambda_{k}^{m_{k}}}\right|_{\lambda_{1}=\lambda_{2}=\cdots=\lambda_{n}=0}
$$

где

$$
n_{k}=\sum_{j=1}^{k} m_{j}
$$

Для гауссовского случайного процесса с $\langle\eta(t)\rangle=0$ характеристическая функция

$$
\Phi_{n}\{\lambda\}=\exp \left\{-\frac{1}{2} \sum_{k, m=1}^{n} \lambda_{k} C_{k m} \lambda_{m}\right\}
$$


где $C_{k m}=\left\langle\eta\left(t_{k}\right) \eta\left(t_{m}\right)\right\rangle$ - элементы корреляционной матрицы $\widehat{C}$ [8]. Соответственно корреляционные функции произведений нечетного числа решеточных переменных равны нулю, а четного числа представимы, как следует из (3) и (4), в виде сумм произведений парных корреляционных функций, полученных всевозможными спариваниями [9]:

$$
\left\langle\prod_{k=1}^{2 n} \eta\left(t_{k}\right)\right\rangle=\sum_{k=2}^{2 n}\left\langle\eta\left(t_{1}\right) \eta\left(t_{k}\right)\right\rangle\left\langle\eta\left(t_{2}\right) \ldots \eta\left(t_{k-1}\right) \eta\left(t_{k+1}\right) \ldots \eta\left(t_{2 n}\right)\right\rangle .
$$

Поскольку корреляционная функция $n$-го слагаемого в (2) содержит сумму произведений случайных величин вида

$$
\sum_{m=0}^{n}\left(\begin{array}{c}
n \\
m
\end{array}\right) \Omega_{1}^{m} \Omega_{2}^{n-m} \prod_{k=1}^{m} \eta\left(t_{k}\right) \prod_{l=m+1}^{n} \eta\left(t_{l}\right)^{2},
$$

то при графическом отображении процедуры спаривания случайную величину из первого произведения можно представить одновалентной вершиной, из второго произведения - двухвалентной [10], а парные корреляционные функции - соединяюшими эти вершины ребрами. Связанная часть всех получаемых графов равна [11]

$$
\frac{1}{2}\left[n ! C_{n}+(n-1) ! O_{n}\right]
$$

где

$$
C_{n}=\Omega_{1}^{2} \Omega_{2}^{n-2} \prod_{k=1}^{n}\left\{\int_{0}^{t} d t_{k}\right\}\left\langle\eta\left(t_{1}\right) \eta\left(t_{2}\right)\right\rangle\left\langle\eta\left(t_{2}\right) \eta\left(t_{3}\right)\right\rangle \ldots\left\langle\eta\left(t_{n-1}\right) \eta\left(t_{n}\right)\right\rangle
$$

- цепочка $n$-го порядка,

$$
O_{n}=\Omega_{2}^{n} \prod_{k=1}^{n}\left\{\int_{0}^{t} d t_{k}\right\}\left\langle\eta\left(t_{1}\right) \eta\left(t_{2}\right)\right\rangle\left\langle\eta\left(t_{2}\right) \eta\left(t_{3}\right)\right\rangle \ldots\left\langle\eta\left(t_{n}\right) \eta\left(t_{1}\right)\right\rangle
$$

- петля $n$-го порядка. Название каждой отдельной диаграммы обусловлено совпадением или несовпадением начального и конечного моментов времени в последовательности перемножаемых корреляционных функций. Если петли порождены исключительно квадратичным вкладом флуктуаций, то цепочки начиная с $n=3$ порождены совместно и квадратичным, и линейным вкладами флуктуаций. Цепочка с $n=2$ обусловлена исключительно линейным вкладом флуктуаций.

С помошью (5) сумму всех связанных и несвязанных диаграмм в (2) можно представить согласно теореме Майера [12] в виде

$$
G(t)=\exp \left\{-i \Omega_{0} t+F_{C}(t)+F_{O}(t)\right\}
$$

где

$$
F_{C}(t)=\sum_{n=2}^{\infty} \frac{(-i)^{n}}{2} C_{n}, \quad F_{O}(t)=\sum_{n=1}^{\infty} \frac{(-i)^{n}}{2 n} O_{n}
$$


Из этого соотношения при $\Omega_{2}=0$ следует известное выражение [1-3]

$$
G(t)=\exp \left\{-i \Omega_{0} t-\Omega_{1}^{2} \int_{0}^{t}\left(t-t_{1}\right)\left\langle\eta\left(t_{1}\right) \eta(0)\right\rangle d t_{1}\right\}
$$

Рассмотрим вспомогательный ряд

$$
\begin{aligned}
R\left(t_{a}, t_{b} \mid t\right)= & \left\langle\eta\left(t_{a}\right) \eta\left(t_{b}\right)\right\rangle+ \\
& +\sum_{n=1}^{\infty}\left(-i \Omega_{2}\right)^{n} \prod_{k=1}^{n}\left\{\int_{0}^{t} d t_{k}\right\}\left\langle\eta\left(t_{a}\right) \eta\left(t_{1}\right)\right\rangle\left\langle\eta\left(t_{1}\right) \eta\left(t_{2}\right)\right\rangle \ldots\left\langle\eta\left(t_{n}\right) \eta\left(t_{b}\right)\right\rangle,
\end{aligned}
$$

позволяющий рассчитать вклады цепочек и петель через соотношения

$$
F_{C}(t)=-\frac{\Omega_{1}^{2}}{2} \int_{0}^{t} \int_{0}^{t} R\left(t_{1}, t_{2} \mid t\right) d t_{2} d t_{1}, \quad F_{O}(t)=-\frac{i \Omega_{2}}{2} \int_{0}^{t} R\left(t_{1}, t_{1} \mid t_{1}\right) d t_{1} .
$$

Из определения этого ряда следует, что он подчиняется уравнению

$$
R\left(t_{a}, t_{b} \mid t\right)+i \Omega_{2} \int_{0}^{t}\left\langle\eta\left(t_{a}\right) \eta\left(t_{1}\right)\right\rangle R\left(t_{1}, t_{b} \mid t\right) d t_{1}=\left\langle\eta\left(t_{a}\right) \eta\left(t_{b}\right)\right\rangle
$$

или

$$
R\left(t_{a}, t_{b} \mid t\right)+i \Omega_{2} \int_{0}^{t} R\left(t_{a}, t_{1} \mid t\right)\left\langle\eta\left(t_{1}\right) \eta\left(t_{b}\right)\right\rangle d t_{1}=\left\langle\eta\left(t_{a}\right) \eta\left(t_{b}\right)\right\rangle .
$$

Равенство

$$
\frac{1}{2 \pi} \int_{-\infty}^{\infty} f(\omega) \exp \left\{i \omega\left(t_{a}-t_{b}\right)\right\} d \omega=f\left(-i \frac{\partial}{\partial t_{a}}\right) \delta\left(t_{a}-t_{b}\right)
$$

$(\delta(t)$ - функция Дирака) позволяет для стационарного случайного процесса преобразовать интегральное уравнение (8a) в дифференциальное

$$
\begin{gathered}
R\left(t_{a}, t_{b} \mid t\right)+i \Omega_{2} K\left(-i \frac{\partial}{\partial t_{a}}\right) \int_{0}^{t} \delta\left(t_{a}-t_{1}\right) R\left(t_{1}, t_{b} \mid t\right) d t_{1}= \\
=\left[1+i \Omega_{2} K\left(-i \frac{\partial}{\partial t_{a}}\right)\right] R\left(t_{a}, t_{b} \mid t\right)=\left\langle\eta\left(t_{a}\right) \eta\left(t_{b}\right)\right\rangle
\end{gathered}
$$

и уравнение (8б) в дифференциальное уравнение

$$
\left[1+i \Omega_{2} K\left(i \frac{\partial}{\partial t_{b}}\right)\right] R\left(t_{a}, t_{b} \mid t\right)=\left\langle\eta\left(t_{a}\right) \eta\left(t_{b}\right)\right\rangle
$$

где $K(\omega)$ - фурье-образ решеточной корреляционной функции $\langle\eta(t) \eta(0)\rangle$ и учтено, что $\left\{t_{a}, t_{b}\right\} \in(0, t)$. 
Если недифференцируемую переменную рассматривать как параметр, а по дифференцируемой совершить прямое и затем обратное преобразования Фурье, то решение уравнений (9) можно получить в виде

$$
\begin{aligned}
R\left(t_{a}, t_{b} \mid t\right)= & \frac{1}{2 \pi} \int_{-\infty}^{\infty} \frac{K(\omega)}{1+i \Omega_{2} K(\omega)} \exp \left\{i \omega\left(t_{a}-t_{b}\right)\right\} d \omega+ \\
& +\sum_{m, n} a_{m n} \exp \left\{i\left(\omega_{m} t_{a}-\omega_{n} t_{b}\right)\right\}= \\
= & \sum_{m} k_{m} \exp \left\{i \omega_{m}\left(t_{a}-t_{b}\right)\right\}+\sum_{m, n} a_{m n} \exp \left\{i\left(\omega_{m} t_{a}-\omega_{n} t_{b}\right)\right\},
\end{aligned}
$$

где $\omega_{m}$ и $\omega_{n}-$ корни характеристического уравнения

$$
1+i \Omega_{2} K(\omega)=0, \quad k_{m}=i \operatorname{Res}\left\{\frac{K\left(\omega_{m}\right)}{1+i \Omega_{2} K\left(\omega_{m}\right)}\right\} \theta\left\{\operatorname{Im}\left[\omega_{m}\left(t_{a}-t_{b}\right)\right]\right\},
$$

функция Хевисайда $\theta(x)$ используется для исключения неохваченных контуром интегрирования в (10) полюсов подынтегральной функции, коэффициенты $a_{m n}$ определяются подстановкой (10) в уравнения (8), форма решения однородной части уравнений (9) приведена для случая некратных корней.

\section{2. ФУНКЦИЯ РЕЛАКСАЦИИ И ГАУСС-МАРКОВСКИЙ ПРОЦЕСС}

В случае гаусс-гауссовского процесса, $\langle\eta(t) \eta(0)\rangle=\exp \left(-\beta t^{2}\right)$, у характеристического уравнения имеется бесконечное число корней, а ряд (10) не удается просуммировать. Решение упрощается, если $K(\omega)$ является рациональной дробью. Например, для многоэкспоненциального гаусс-марковского процесса

$$
\langle\eta(t) \eta(0)\rangle=\sum_{n=1}^{N} p_{n} \exp \left(-\alpha_{n}|t|\right)
$$

Четность

$$
K(\omega)=2 \sum_{n=1}^{N} \frac{p_{n} \alpha_{n}}{\alpha_{n}^{2}+\omega^{2}}
$$

по $\omega$ позволяет для случая некратных корней характеристического уравнения искать решение уравнений (8) в виде

$$
R\left(t_{a}, t_{b} \mid t\right)=\sum_{m=1}^{N}\left[k_{m} \exp \left(-\lambda_{m}\left|t_{a}-t_{b}\right|\right)+a_{m}^{+} \exp \left(\lambda_{m} t_{a}\right)+a_{m}^{-} \exp \left(-\lambda_{m} t_{a}\right)\right]
$$

где суммирование проводится по решениям характеристического уравнения $1+$ $i \Omega_{2} K(i \lambda)=0$ только с $\operatorname{Re}\{\lambda\}>0$, а коэффициенты частного решения дифференциального уравнения

$$
k_{m}=i \frac{\alpha_{m}^{2}-\lambda_{m}^{2}}{2 \Omega_{2} \lambda_{m}} \prod_{\substack{n=1 \\ n \neq m}}^{N} \frac{\alpha_{n}^{2}-\lambda_{m}^{2}}{\lambda_{n}^{2}-\lambda_{m}^{2}}
$$


Подстановка (11) в уравнения (8) и выравнивание коэффициентов перед экспонентами с равными показателями приводят к системе из $2 N$ линейных уравнений для $\left\{a_{m}^{ \pm}\right\}$

$$
\begin{gathered}
\sum_{m=1}^{N}\left[\frac{k_{m} \exp \left(\lambda_{m}\left(t_{b}-t\right)\right)}{\alpha_{n}+\lambda_{m}}+\frac{a_{m}^{+} \exp \left(\lambda_{m} t\right)}{\alpha_{n}-\lambda_{m}}+\frac{a_{m}^{-} \exp \left(-\lambda_{m} t\right)}{\alpha_{n}+\lambda_{m}}\right]=0, \\
\sum_{m=1}^{N}\left[\frac{k_{m} \exp \left(-\lambda_{m} t_{b}\right)}{\alpha_{n}+\lambda_{m}}+\frac{a_{m}^{+}}{\alpha_{n}+\lambda_{m}}+\frac{a_{m}^{-}}{\alpha_{n}-\lambda_{m}}\right]=0 .
\end{gathered}
$$

Заменой

$$
a_{m}^{ \pm}=\exp \left(\mp \frac{\lambda_{m} t}{2}\right) \frac{b_{m}^{+} \pm b_{m}^{-}}{2}
$$

эта система разделяется на две независимые системы из $N$ уравнений каждая,

$$
\sum_{m=1}^{N} A_{n m}^{ \pm} b_{m}^{ \pm}=d_{n}^{ \pm}
$$

где

$$
\begin{aligned}
A_{n m}^{ \pm} & =\frac{\exp \left(\frac{\lambda_{m} t}{2}\right)}{\alpha_{n}-\lambda_{m}} \pm \frac{\exp \left(-\frac{\lambda_{m} t}{2}\right)}{\alpha_{n}+\lambda_{m}} \\
d_{n}^{ \pm} & =-\sum_{m=1}^{N} \frac{k_{m}}{\alpha_{n}+\lambda_{m}}\left\{\exp \left[\lambda_{m}\left(t_{b}-t\right)\right] \pm \exp \left[-\lambda_{m} t_{b}\right]\right\} .
\end{aligned}
$$

Расчет матрищ $\widehat{B}^{ \pm}$, обратных к $\widehat{A}^{ \pm}$, позволяет представить вспомогательный ряд в виде

$$
\begin{aligned}
& R\left(t_{a}, t_{b} \mid t\right)-\sum_{m=1}^{N} k_{m} \exp \left(-\lambda_{m}\left|t_{a}-t_{b}\right|\right)= \\
& =\sum_{m=1}^{N}\left\{b_{m}^{+} \operatorname{ch}\left[\lambda_{m}\left(t_{a}-\frac{t}{2}\right)\right]+b_{m}^{-} \operatorname{sh}\left[\lambda_{m}\left(t_{a}-\frac{t}{2}\right)\right]\right\}= \\
& =\sum_{m, n=1}^{N}\left\{D_{m n}^{+}(t) \operatorname{ch}\left[\lambda_{m}\left(t_{a}-\frac{t}{2}\right)\right] \operatorname{ch}\left[\lambda_{n}\left(t_{b}-\frac{t}{2}\right)\right]+\right. \\
& \left.\quad+D_{m n}^{-}(t) \operatorname{sh}\left[\lambda_{m}\left(t_{a}-\frac{t}{2}\right)\right] \operatorname{sh}\left[\lambda_{n}\left(t_{b}-\frac{t}{2}\right)\right]\right\},
\end{aligned}
$$

где

$$
D_{m n}^{ \pm}(t)=-2 k_{n} \exp \left(-\frac{\lambda_{n} t}{2}\right) \sum_{l=1}^{N} \frac{B_{m l}^{ \pm}(t)}{\alpha_{l}+\lambda_{n}}
$$

6 Теоретическая и математическая физика, т. 121, № 2, 1999 г. 
В соответствии с (7) вклады цепочек и петель представляются выражениями

$$
\begin{aligned}
F_{C}(t)= & -\Omega_{1}^{2}\left[\sum_{m=1}^{N} k_{m} \frac{\lambda_{m} t+\exp \left(-\lambda_{m} t\right)-1}{\lambda_{m}^{2}}+2 \sum_{m, n=1}^{N} D_{m n}^{+}(t) \frac{\operatorname{sh}\left(\frac{\lambda_{m} t}{2}\right) \operatorname{sh}\left(\frac{\lambda_{n} t}{2}\right)}{\lambda_{m} \lambda_{n}}\right], \\
F_{O}(t)= & -\frac{i \Omega_{2}}{2}\left\{t \sum_{m=1}^{N} k_{m}+\sum_{m, n=1}^{N} \int_{0}^{t}\left[D_{m n}^{+}\left(t_{1}\right) \operatorname{ch}\left(\frac{\lambda_{m} t_{1}}{2}\right) \operatorname{ch}\left(\frac{\lambda_{n} t_{1}}{2}\right)+\right.\right. \\
& \left.\left.+D_{m n}^{-}\left(t_{1}\right) \operatorname{sh}\left(\frac{\lambda_{m} t_{1}}{2}\right) \operatorname{sh}\left(\frac{\lambda_{n} t_{1}}{2}\right)\right] d t_{1}\right\} .
\end{aligned}
$$

Приведем решение для одноэкспоненциального процесса $\langle\eta(t) \eta(0)\rangle=p \exp (-\alpha|t|)$ :

$$
\begin{aligned}
R\left(t_{a}, t_{b} \mid t\right)= & \frac{p \alpha}{\lambda}\left(\exp \left[-\lambda\left|t_{a}-t_{b}\right|\right]+\frac{2}{D(t)}\left\{\operatorname{ch}\left[\lambda\left(t_{a}-t_{b}\right)\right]-\right.\right. \\
& \left.\left.-\frac{\alpha+\lambda}{\alpha-\lambda} \operatorname{ch}\left[\lambda\left(t_{a}+t_{b}-t\right)\right] \exp (\lambda t)\right\}\right)
\end{aligned}
$$

где $\lambda=\left[\alpha\left(\alpha+i 2 \Omega_{2} p\right)\right]^{1 / 2}, D(t)=[(\alpha+\lambda) /(\alpha-\lambda)]^{2} \exp (2 \lambda t)-1$. Соответственно для вкладов цепочек и петель $(7)$ имеем

$$
\begin{aligned}
& F_{C}(t)=\frac{\Omega_{1}^{2} p \alpha}{\lambda^{2}}\left[-t+\frac{2 \alpha}{\lambda} \frac{\alpha \operatorname{ch}(\lambda t)+\lambda \operatorname{sh}(\lambda t)-\alpha}{2 \alpha \lambda \operatorname{ch}(\lambda t)+\left(\alpha^{2}+\lambda^{2}\right) \operatorname{sh}(\lambda t)}\right] \\
& F_{O}(t)=\frac{\alpha t}{2}-\frac{1}{2} \ln \left[\operatorname{ch}(\lambda t)+\frac{\alpha^{2}+\lambda^{2}}{2 \alpha \lambda} \operatorname{sh}(\lambda t)\right] .
\end{aligned}
$$

Эти выражения совпадают с выражениями, полученными при $p=1$ методом кинетического уравнения для плотности вероятности [13], что можно считать проверкой развитого в настоящей работе подхода.

\section{3. ФОРМА ЛИНИИ МАГНИТНОГО РЕЗОНАНСА И СКОРОСТЬ ФЛУКТУАЦИЙ}

Компактный анализ воздействия флуктуаций можно провести на форме резонансной линии

$$
g(\omega)=\int_{-\infty}^{\infty} G(t) \exp (i \omega t) d t
$$

с помошью соотношений (12) и (13) при $p=1$. В случае статических флуктуаций $(\alpha=+0)$ резонансная линия представляет собой асимметричное распределение резонансных частот

$$
\begin{aligned}
g(\omega)= & \left\{\frac{8 \pi}{\Omega_{1}^{2}+2 \Omega_{2}\left(\omega-\Omega_{0}\right)}\right\}^{1 / 2} \exp \left\{-\frac{\Omega_{1}^{2}+\Omega_{2}\left(\omega-\Omega_{0}\right)}{\Omega_{2}^{2}}\right\} \times \\
& \times \operatorname{ch}\left\{\frac{\Omega_{1}\left[\Omega_{1}^{2}+2 \Omega_{2}\left(\omega-\Omega_{0}\right)\right]^{1 / 2}}{\Omega_{2}^{2}}\right\}, \quad \Omega_{1}^{2}+2 \Omega_{2}\left(\omega-\Omega_{0}\right)>0, \\
g(\omega)= & 0, \quad \Omega_{1}^{2}+2 \Omega_{2}\left(\omega-\Omega_{0}\right)<0,
\end{aligned}
$$



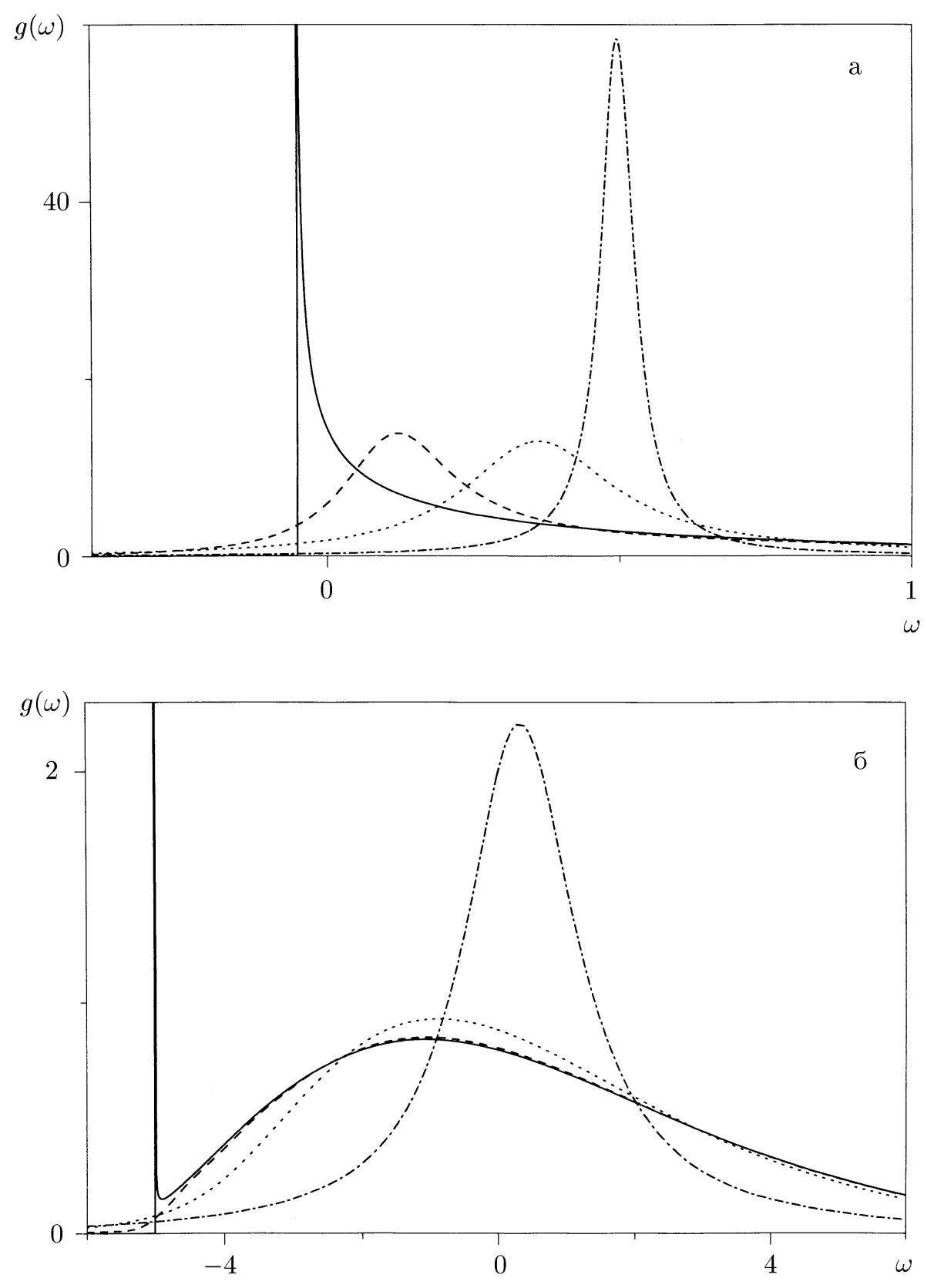

Рис. 1

демонстрируемое сплошной линией на рис. 1 . При расчете были зафиксированы значения $\Omega_{0}=0$ и $\Omega_{2}=1$, а варьировалась величина $\Omega_{1}: 10^{-1 / 2}$ для рис. $1 \mathrm{a}, 10^{1 / 2}$ для рис. 1б. В области высших частот $g(\omega)$ спадает по экспоненшиальному закону (без по- 
тери общности полагаем $\Omega_{1}>0$ и $\Omega_{2}>0$, знак $\Omega_{1}$ не влияет на $g(\omega)$, для $\Omega_{2}<0$ резонансную линию можно получить симметричным отражением линии, анализируемой для случая $\Omega_{2}>0$ относительно частоты $\Omega_{0}$ ), переходяшему в гауссовский спад при $\Omega_{2}=+0$. В области низших частот резонансная линия оканчивается сингулярностью корневого типа в точке $\omega=\Omega_{0}-\Omega_{1}^{2} / 2 \Omega_{2}$. При $\Omega_{2} \ll \Omega_{1}$ корневая сингулярность уходит далеко в область отрицательных частот и ее интегральньй вес спадает по экспоненциальному закону, а в центре спектра форма линии

$$
g(\omega) \approx\left\{\frac{2 \pi}{\Omega_{1}^{2}+2 \Omega_{2}\left(\omega-\Omega_{0}\right)}\right\}^{1 / 2} \exp \left\{-\frac{\left(\omega-\Omega_{0}\right)^{2}}{2\left[\Omega_{1}^{2}+\Omega_{2}\left(\omega-\Omega_{0}\right)\right]}\right\}
$$

близка к гауссовской, но с асимметрией и сдвигом максимума к точке $\omega \approx \Omega_{0}-\Omega_{2}$. Для наблюдения такой трансформации спектра необходимо варьировать как величину, так и направление постоянного магнитного поля относительно кристаллографических осей исследуемого образца.

Динамические флуктуации существенно изменяют форму резонансной линии, как это видно на рис. 1 для различных $\alpha>0$ : $10^{-1}$ - штриховая линия, 1 - пунктирная линия, 10 - штрих-пунктирная линия. В первую очередь флуктуации подавляют корневую сингулярность, тем самым уширяя спектр. И лишь затем с ростом скорости $\alpha$ проявляется их сужающее действие $[1,3]$, приводящее и к перемешению пика к средней частоте

$$
\langle\omega\rangle=(2 \pi)^{-1} \int_{-\infty}^{\infty} \omega g(\omega) d \omega=\Omega_{0}+\frac{\Omega_{2}}{2} .
$$

Быстрым по сравнению с $\Omega_{2}$ флуктуациям $\left(\Omega_{2} / \alpha=+0\right)$ отвечает симметричная резонансная линия, описываемая известным выражением (6).

Такое поведение шшиины линии можно понять из оценки $\operatorname{Re}\left\{-\left[F_{C}(t)+F_{O}(t)\right] / t\right\}$ при $t \gg \alpha^{-1}$. Вклады цепочек, $\Omega_{1}^{2} \alpha /\left(\alpha^{2}+4 \Omega_{2}^{2}\right)$, и петель,

$$
\frac{\alpha}{2}\left[\sqrt{\frac{1}{2}\left(1+\sqrt{1+\frac{4 \Omega_{2}^{2}}{\alpha^{2}}}\right)}-1\right],
$$

являются немонотонными функциями скорости флуктуаций, сначала растушими с ростом $\alpha$, а затем спадаюшими. Из оценки же $\operatorname{Im}\left\{-\left[F_{C}(t)+F_{O}(t)\right] / t\right\}$ при $t \gg \alpha^{-1}$ следует, что вклады цепочек, $-\Omega_{1}^{2} \Omega_{2} /\left(\alpha^{2}+4 \Omega_{2}^{2}\right)$, и петель,

$$
\frac{\Omega_{2}}{2 \sqrt{\frac{1}{2}\left(1+\sqrt{1+\frac{4 \Omega_{2}^{2}}{\alpha^{2}}}\right)}}
$$

в сдвиг максимума линии являются монотонными функциями $\alpha$.

В целом спектр магнитного резонанса немонотонно зависит от квадратичного вклада флуктуаций решеточной системы в резонансную частоту. Замедление флуктуаций приводит к смешению и асимметрии резонансной линии и в однородном постоянном магнитном поле. Численное решение уравнения (8) для гаусс-гауссовского процесса выявило те же закономерности. 


\section{4. РЕЗОНАНСНАЯ ЛИНИЯ И НЕПРЕРЫВНЫЙ ФАЗОВЫЙ ПЕРЕХОД В КРИСТАЛЛЕ С РЕЛАКСАЦИОННЫМ ТИПОМ ДИНАМИКИ}

Замедление флуктуаций характерно для окрестности непрерывного фазового перехода [14]. За исключением узкой окрестности температуры перехода $\left|T-T_{\mathrm{c}}\right| / T_{\mathrm{c}}<10^{-4}$ $10^{-3}[15]$, поведение решеточной системы успешно описывается термодинамической теорией Ландау, позволяюшей рассматривать движение атомов как стационарный гауссовский случайный процесс. Для его описания воспользуемся простейшей формой неполного термодинамического потенциала [14]

$$
\Phi\left[\eta_{r}\right]=\Phi_{0}+\int\left\{\frac{A \eta_{r}^{2}}{2}+\frac{B \eta_{r}^{4}}{4}+\frac{D\left(\nabla \eta_{r}\right)^{2}}{2}\right\} d r
$$

где $A=a\left(T-T_{\mathrm{c}}\right), a>0, B>0, D>0, \nabla$-набла-оператор.

Атомы хаотически движутся относительно положений равновесия

$$
\left\langle\eta_{r}\right\rangle=\operatorname{Re}\left\{\left(-\frac{A}{B}\right)^{1 / 2}\right\} \equiv \eta_{0},
$$

определяемых из условия

$$
\frac{\delta \Phi}{\delta \eta_{r}}=0
$$

С помощью уравнения движения

$$
\frac{d}{d t} \frac{\delta \Phi}{\delta \dot{\eta}_{r}}=-\frac{\delta \Phi}{\delta \eta_{r}}-\frac{\delta \Psi}{\delta \dot{\eta}_{r}}
$$

$\left(\dot{\eta}=d \eta / d t, \Psi\left[\dot{\eta}_{r}\right]=\int\left\{\Gamma \dot{\eta}_{r}^{2} / 2\right\} d r\right.$ - диссипативная функция) и диссипативно-флуктуационной теоремы [14] можно найти корреляционную функцию для флуктуаций $\eta_{r}^{\prime}(t)=$ $\eta_{r}(t)-\eta_{0}$ решетки с релаксационным типом динамики $\left(\delta \Phi / \delta \dot{\eta}_{r} \approx 0\right)$ :

$$
\left\langle\eta_{r}^{\prime}(t) \eta_{r}^{\prime}(0)\right\rangle=\frac{1}{\pi} \int_{0}^{\infty}\left\langle\eta_{r}^{\prime 2}\right\rangle_{\omega} \cos (\omega t) d \omega, \quad\left\langle\eta_{r}^{\prime 2}\right\rangle_{\omega}=\frac{v}{8 \pi^{3}} \int \frac{T \Gamma d k}{A_{k}^{2}+\Gamma^{2} \omega^{2}},
$$

где $A_{k}=A+3 B \eta_{0}^{2}+D k^{2}, v$ - объем элементарной ячейки в неупорядоченной фазе, интегрирование по волновым векторам $k$ производится внутри первой зоны Бриллюэна, константа Больцмана полагается равной единице.

Форма корреляционной функции после интегрирования по частоте

$$
\left\langle\eta_{r}^{\prime}(t) \eta_{r}^{\prime}(0)\right\rangle=\frac{v T}{16 \pi^{3}} \int \frac{\exp \left(-\frac{A_{k}|t|}{\Gamma}\right)}{A_{k}} d k
$$

указывает на то, что движение атомов в данном случае описывается многоэкспоненциальным гаусс-марковским процессом с дисперсией скоростей флуктуаций $\alpha_{k}=A_{k} / \Gamma$. Решение уравнений (8) приведенным в разделе 2 аналитическим методом из-за того, что 

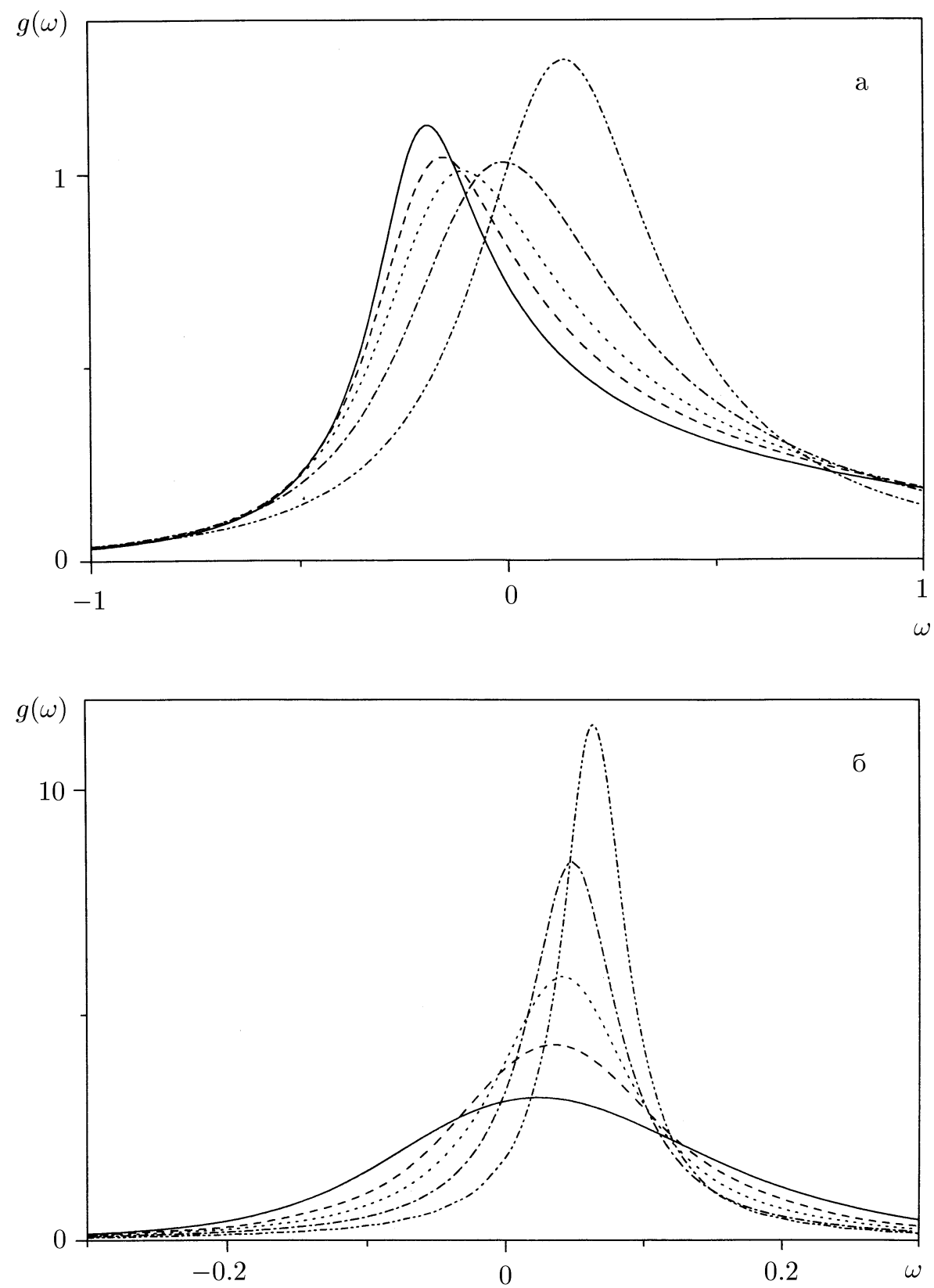

Рис. 2

$N \rightarrow \infty$, становится невозможным. Поэтому обратимся к результатам численного моделирования.

На рис. 2 приведены резонансные линии, рассчитанные для неупорядоченной фазы $\eta_{0}=0$. Были зафиксированы следуюшие величины: $\Omega(\eta)=\eta+\eta^{2} / 2, T_{\mathrm{c}}=v=a=$ 
$\Gamma=1, Q=\left(6 \pi^{2} / v\right)^{1 / 3}$ - дебаевский волновой вектор. Варьировалась величина $D$ : $10^{-1}$ для рис. $2 \mathrm{a}, 1$ - для рис. 26 . Температурному отклонению от точки фазового пере-

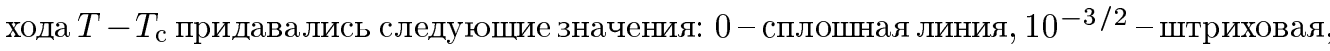
$10^{-1}$ - пунктирная, $10^{-1 / 2}$ - штрих-пунктирная, 1 - штриховая с двойным пунктиром. Отличие формы резонансных линий в упорядоченной фазе $\left(\eta_{0} \neq 0\right)$ сводится к сдвигу по частоте вследствие того, что $\Omega_{0}=\Omega\left(\eta_{0}\right)$, и относительному росту линейного вклада флуктуаций в результате того, что $\Omega_{1}=\partial \Omega\left(\eta_{0}\right) / \partial \eta_{0}$.

$\mathrm{B}$ целом температурное развитие асимметрии линии, ее смешение и изменение ширины в пределах каждой из фаз отвечают представлениям, полученным в предыдушем разделе. Статическими в точке $T_{\mathrm{c}}$ остаются только флуктуации с $k=0$, а остальные уменьшают температурную область сужения линии в окрестности точки фазового перехода вплоть до ее исчезновения с ростом $D$, тем самым восстанавливая типичную для случая линейного вклада флуктуаций в резонансную частоту монотонность роста ширины линии при приближении к значению $T_{\mathrm{c}}$.

Такое поведение резонансной линии можно понять и с помошью оценки вкладов цепочек и петель на больших временах:

$$
C_{n} \approx t \Omega_{1}^{2} \Omega_{2}^{n-2}\left\langle\eta_{r}^{\prime 2}\right\rangle_{0}^{n-1}, \quad O_{n} \approx \frac{t \Omega_{2}^{n}}{\pi} \int_{0}^{\infty}\left\langle\eta_{r}^{\prime 2}\right\rangle_{\omega}^{n} d \omega
$$

При оценке $C_{n}$ учтено, что в спектре колебаний “мягкой” решеточной системы при $T \neq$ $T_{\mathrm{c}}$ присутствует щель. В результате получаем

$$
\begin{aligned}
& F_{C}(t) \approx-\Delta_{C} t, \quad \Delta_{C}=\frac{\Omega_{1}^{2}}{2\left(\left\langle\eta_{r}^{\prime 2}\right\rangle_{0}^{-1}+i \Omega_{2}\right)}, \\
& F_{O}(t) \approx-\Delta_{O} t, \quad \Delta_{O}=\frac{1}{2 \pi} \int_{0}^{\infty} \ln \left(1+i \Omega_{2}\left\langle\eta_{r}^{\prime 2}\right\rangle_{\omega}\right) d \omega .
\end{aligned}
$$

В отличие от случая линейного вклада флуктуаций, вклад цепочек в ширину линии $\operatorname{Re}\left\{\Delta_{C}\right\}$ исчезает при приближении к $T_{\mathrm{c}}$ из-за неограниченного роста $\left\langle\eta_{r}^{\prime 2}\right\rangle_{0}$. В то же время вкладпетель остается отличным от нуля: $\Delta_{O}=\left(\lambda-\alpha_{0}\right) / 2, \lambda=\left(\alpha_{0}^{2}+i \Omega_{2} T / \Gamma\right)^{1 / 2}$ при $D=0$. С ростом $D$ корреляционные функции $\left\langle\eta_{r}^{\prime 2}\right\rangle_{\omega}$ спадают согласно (14), что приводит к ослаблению выявленных в предыдушем разделе эффектов и уменьшению температурной области их возможного наблюдения.

Особенности развития резонансной линии при приближении к точке фазового перехода благодаря квадратичному вкладу решеточных флуктуаций в резонансную частоту спиновой системы позволяют по-иному взглянуть на экспериментальные результаты работ, в которых появление асимметрии линии интерпретировалось как проявление предшественников перехода - долгоживущих кластеров последуюшей фазы [16].

Благодарности. Авторы признательны В.Е. Зобову за полезные обсуждения. 


\section{Список литературы}

[1] А. Абрагам. Ядерный магнетизм. М.: ИЛ, 1963.

[2] P. W. Anderson, P. R. Weiss. Rev. Mod. Phys. 1953. V. 25. P. 269.

[3] P. W. Anderson. J. Phys. Soc. Japan. 1954. V. 9. P. 316.

[4] W. B. Mims. Phys. Rev. 1968. V. 168. P. 370.

[5] В.Е, Зобов, А. А. Лундин. Письма в ЖЭТФ. 1986. Т. 43. С. 418.

[6] Э. Л. Нагаев. Магнетики со сложными обменными взаимодействиями. М.: Наука, 1988.

[7] У. Хеберлен, М. Меринг. ЯМР высокого разрешения в твердых телах. М.: Мир, 1980; U. Haeberlen. High Resolution NMR in Solids: Selective Averaging. New York-San Francisco-London: Academic Press, 1976; M. Mehring. High Resolution in NMR Spectroscopy in Solids. Berlin-Heidelberg-New York: Springer-Verlag, 1976.

[8] И. Н. Коваленко, Н. Ю. Кузнецов, В. М. Шуренков. Случайные процессы. Киев: Наукова Думка, 1983.

[9] R. Kubo, M. Toda, N. Hashitsume. Statistical physics. II. Berlin-Heidelberg: Springer-Verlag, 1985.

[10] В. А. Емеличев, О.И. Мельников, В.И. Сарванов, Р. И. Тышкевич. Лекции по теории графов. М.: Наука, 1990.

[11] В. Е. Зобов, М. А. Попов. ТМФ. 1995. Т. 102. С. 305.

[12] Дж. Уленбек, Джс. Форд. Лекции по статистической механике. М.: Мир, 1965.

[13] J. Euczka. J. Phys. A. 1988. V. 21. P. 3063.

[14] Л. Д. Ландау, Е. М. Лифиич. Статистическая физика. Часть 1. М.: Наука, 1976.

[15] В. Л. Гинзбург. ФТТ. 1960. Т. 2. С. 2031.

[16] R. L. Amstrong. Progr. in NMR Spectroscopy. 1989. V. 21. P. 151.

Поступила в редакцию 18.I.1999 г., после доработки 13.IV.1999 г. 